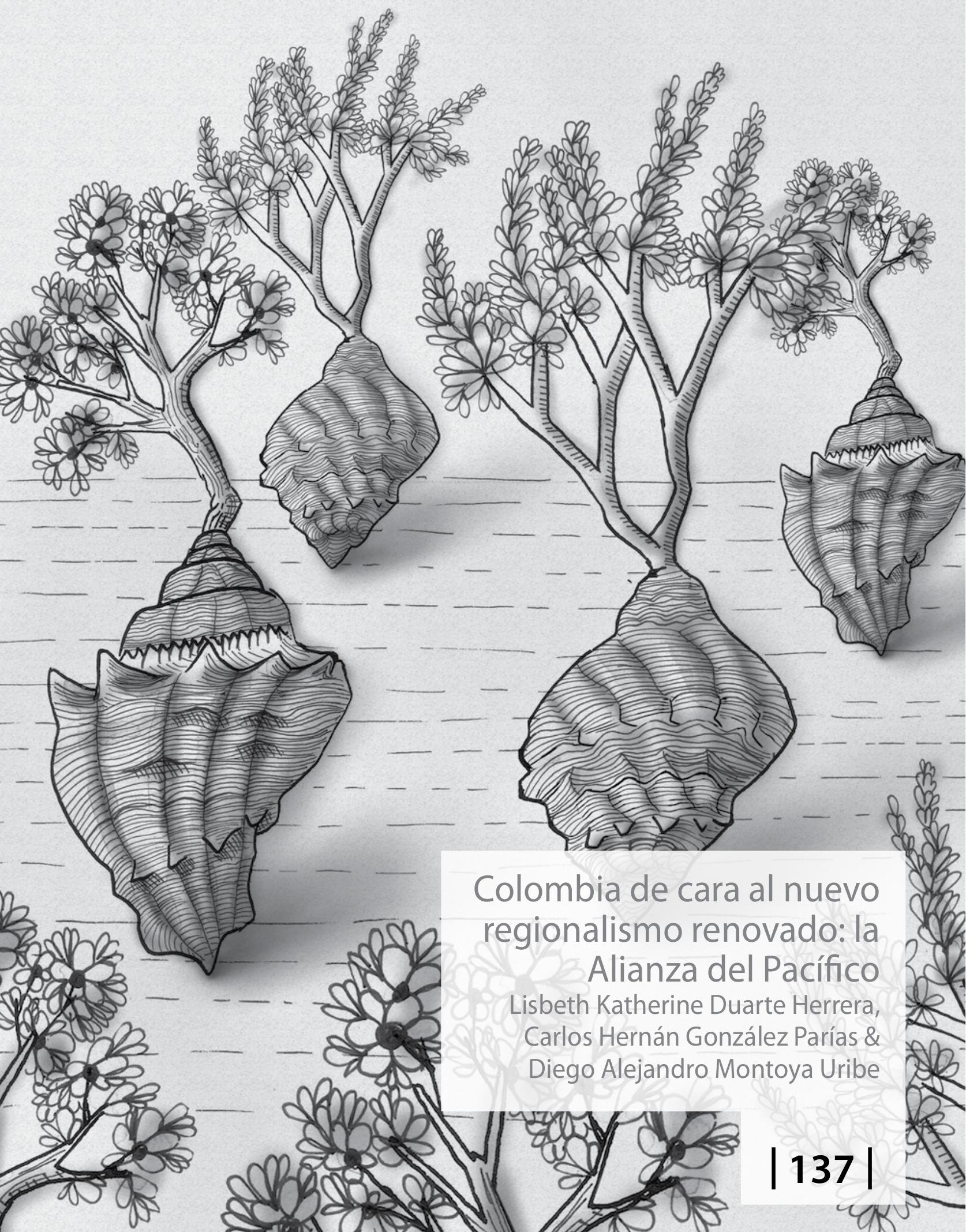





\title{
Colombia de cara al nuevo regionalismo renovado: la Alianza del Pacífico ${ }^{1}$ \\ Colombia in View of the Renewed Regionalism: The Pacific Alliance
}

Recibido: 12 de marzo de 2015

Evaluado: 26 de marzo de 2015

Aceptado: 29 de marzo de 2015

\author{
Lisbeth Katherine Duarte Herrera (Colombia) \\ lisbeth.duarte@esumer.edu.co \\ Politóloga Universidad Nacional de Colombia y Magister en Ciencias Sociales \\ con especialidad en Desarrollo municipal El Colegio Mexiquense A.C. \\ Institución Universitaria Esumer
}

\section{Carlos Hernán González Parías (Colombia)}

carlos.gonzalez88@esumer.edu.co

Politólogo Universidad Nacional de Colombia y Magister en Gobierno

Universidad de Medellín

Institución Universitaria Esumer

\section{Diego Alejandro Montoya Uribe (Colombia) \\ diego.montoya@esumer.edu.co \\ Negociador Internacional Universidad EAFIT y Magister en Comercio y \\ Relaciones Internacionales Universidad Paris 12 \\ Istitución Universitaria Esumer}

\section{Resumen}

La Alianza del Pacífico se presenta como un mecanismo de integración regional novedoso, ajustado a ciertas particularidades históricas de la región y de los Estados fundadores. Pero también, como proceso de integración comparte características con ciertas formas del regionalismo, especialmente con el regionalismo abierto característico de los años 90 del siglo XX, por lo cual, la Alianza del Pacífico se presenta como un nuevo regionalismo renovado, actualizado y autodenominado de integración profunda. La internacionalización económica por medio de inserción a nuevos mercados, es uno de los elementos que comparten los cuatro Estados fundadores: México, Colombia, Perú y Chile. En su conjunto estos países acaparan

\begin{abstract}
The Pacific Alliance is an original integration mechanism adjusted to specific historical peculiarities of the region and of the founding states. It also shares some features with certain regionalism indications, especially with the open regionalism that characterized the 90 s of the $X X$ century. For this reason, The Pacific Alliance is presented as a renewed and updated regionalism, which is considered of deep integration. Economic internationalization through the incorporation to new markets is one of the elements shared by the four founding states: Mexico, Colombia, Peru, and Chile. These countries control more than half of the Latin American foreign trade. Nevertheless,
\end{abstract}

1 Este texto presenta los resultados parciales de la investigación: Observatorio de Comercio Exterior Colombiano, realizada en el año 2014 por el Grupo de Investigación en Estudios Internacionales de la Institución Universitaria Esumer. 
más de la mitad del comercio exterior latinoamericano. No obstante, analizando en detalle se observa que países como Perú y especialmente Colombia, se encuentran un poco rezagados en dicha materia con respecto a sus similares de México y Chile, incluso si se mira a otros países de la región. Los acuerdos comerciales y en especial la Alianza del Pacífico se presenta como una importante oportunidad en la estrategia colombiana de internacionalización económica, pero se debe superar el aun aislamiento y dependencia mono exportadora del país, con el fin de maximizar los beneficios potenciales que estos presentan, ya que se evidencia un importante rezago de Colombia en términos comerciales con respecto a los demás miembros de la alianza, especialmente frente a México y Chile.

Palabras clave: Integración regional, Alianza del Pacífico, indicadores, comercio exterior. by carefully analyzing this matter, it is possible to observe that countries such as Peru and Colombia are left behind with respect to Mexico and Chile, even when compared to other countries of the region. Commercial agreements -especially The Pacific Alliance- are presented as an important opportunity for the Colombian strategy to reach economic internationalization, but it is important to overcome the exporting isolation and dependence of the country in order to maximize its potential benefits. It was evidenced that Colombia is commercially left behind when compared to the other members of the Alliance, especially Mexico and Chile.

Keywords: Regional integration, Pacific Alliance, indicators, foreign trade. 


\section{Introducción}

La Alianza del Pacífico, en adelante A.P., es una iniciativa de integración regional creada en el 2011 por Chile, Colombia, México y Perú.

A pesar de ser una iniciativa de integración relativamente reciente la A.P. pretende ser motor de la integración de América Latina. En consecuencia, ésta ya cuenta con resultados significativos, dentro de los que se encuentran la eliminación de los requisitos de visas de turismo y negocios, así como también, la implementación de programas especiales para facilitar el intercambio cultural y académico para los ciudadanos de los cuatro países miembros. Del mismo modo, a través de un nuevo tratado de libre comercio se pretende eliminar inmediatamente las tarifas para el $92 \%$ de los productos en común de los cuatro países miembros y la eliminación gradual del $8 \%$ restante. Con el fin de promover la libre circulación de capitales, las bolsas de valores de los cuatro países miembros fueron unificadas en el Mercado Integrado Latinoamericano (MILA) buscando diversificar los productos financieros a ofrecer.

Cabe resaltar cómo la Alianza del Pacífico, en su poco tiempo de existencia, ha despertado el interés de otros países. Es así, como a la fecha, la A.P. cuenta con 32 países como miembros observadores procedentes de los cinco continentes, algunos de ellos; Australia, Finlandia, India, Israel, Estados Unidos, entre otros. La alianza busca fortalecer las relaciones con estos países observadores a través de proyectos de gran impacto en materia de educación, comercio, innovación, ciencia y tecnología, infraestructura y fortalecimiento del capital empresarial.

Otro de los resultados que se han logrado materializar en el poco tiempo es el establecimiento de embajadas y oficinas comerciales en instalaciones compartidas en el extranjero, la organización de misiones económicas y comerciales conjuntas, así como también, la creación de un fondo común para promover proyectos tanto entre los cuatro países miembros como con terceros Estados

En conjunto, los cuatro países miembros de la A.P. constituyen un mercado de 214 millones de personas con un PIB total de 2.1 billones de dólares. Esto representa 37\% del total del PIB de América Latina, teniendo en los últimos cuatro años un promedio de crecimiento anual del 5,1\%. El comercio exterior de esta iniciativa de integración genera más de 1.13 billones de dólares y los cuatro países reciben $45 \%$ del total del flujo de inversión extranjera directa de toda América Latina.

Hay tres aspectos importantes del orden económico-comercial característicos de los cuatro países miembros de la Alianza, a saber: 1) poseen políticas económicas neoliberales que propenden por el libre tránsito de personas, bienes, servicios y capital; 2) reconocen la importancia de la región AsiaPacífico y buscan construir un puente con esta región que les permita penetrar dichos mercados; y finalmente, 3) poseen indicadores macroeconómicos estables y ritmos de crecimiento por encima del promedio mundial y regional en los últimos años.

En este sentido, este artículo busca realizar un análisis exploratorio del aspecto económico-comercial de la A.P. basado principalmente en indicadores comerciales haciendo particular énfasis en la situación de Colombia en el contexto de esta iniciativa de integración.

La metodología utilizada en este artículo fue principalmente la tabulación y el análisis de datos de fuentes secundarias como: organizaciones internacionales y agencias oficiales para cada uno de los 
países miembros, con el fin de brindarle veracidad y contrarrestar la información recolectada con el fin de construir tablas e indicadores pertinentes para el análisis en cuestión.

\section{Marco teórico y conceptual para entender la Alianza del Pacífico como proceso de integración}

En cerca de cinco décadas de historia de los procesos regionales de integración en América Latina, éstos se han caracterizado por ser dinámicos y por responder a los diferentes contextos históricos, sociales y políticos particulares. A lo largo de dicha historia, se suelen reconocer diferentes etapas en las cuales se acostumbran a ubicar, según sus características generales, los diferentes esquemas regionales de integración existentes. Por lo tanto, y con la finalidad de brindar un soporte tanto histórico como teórico y conceptual, el presente apartado brinda un rápido pero minucioso recorrido por las diferentes etapas históricas de los procesos de integración, con la pretensión de ubicar teóricamente a la Alianza del Pacífico.

\section{Regionalismo: viejo, nuevo y post-liberal}

El adjetivo nuevo, indica la existencia de un regionalismo previo; el denominado viejo regionalismo. Los estudios referentes a los procesos regionales de integración permiten hacer una primera distinción entre estos; viejo y nuevo regionalismo, lo anterior con el fin de realizar no sólo una distinción temporal de su existencia, sino también, contextual acerca de la forma de proceder y entender los esquemas de integración por medio de los fines y mecanismos empleados.

Se ubica como punto de inflexión del viejo al nuevo regionalismo, el año 1989 (De Lombaerde, Kochi, \& Briceño, 2008, pág. 4) por los sucesos y reconfiguración de poderes que trajo consigo tanto la caída del muro de Berlín como la posterior disolución de la Unión Soviética y por ende el fin del sistema bipolar. Así por ejemplo, Hettne y Söderbaum, señalan que la transformación de un sistema bipolar a uno de estructura multipolar o tripolar con una nueva división de poder y del esquema de trabajo; el declive de la posición hegemónica de Estados Unidos; la caída del sistema Westfaliano del Estado-Nación a favor de una mayor interdependencia y globalización y, el cambio de actitud hacia un desarrollo económico neoliberal y un sistema político asociado en los países desarrollados, así como en los países pertenecientes al antiguo telón de acero (Hettne $\&$ Söderbaum, 2000) fueron hitos o elementos que marcaron claramente un salto en los procesos de integración.

A continuación algunos aspectos que permiten contrastar las diferencias sobresalientes entre viejo y nuevo regionalismo. 
Tabla 1. Características del viejo y nuevo regionalismo

\begin{tabular}{|c|c|c|}
\hline Características & Viejo Regionalismo & Nuevo Regionalismo \\
\hline Contexto histórico & $\begin{array}{l}\text { Se configuró durante y a causa de la } \\
\text { guerra fría: (contexto bipolar). }\end{array}$ & $\begin{array}{l}\text { Surge en buena medida por el fin } \\
\text { de la confrontación bipolar, en el } \\
\text { marco de un orden internacional en } \\
\text { transición hacia una multipolaridad } \\
\text { y del surgimiento de liderazgos } \\
\text { regionales (contexto de } \\
\text { globalización). }\end{array}$ \\
\hline Ejercicio de poder & $\begin{array}{l}\text { Fue creado "desde arriba" liderado } \\
\text { por las súper potencias en la } \\
\text { contienda bipolar para asegurar } \\
\text { zonas de influencia. Procesos Norte- } \\
\text { Sur verticales. }\end{array}$ & $\begin{array}{l}\text { Proceso gestado "desde adentro" } \\
\text { y/o "desde abajo", con participación } \\
\text { activa de Estados periféricos. } \\
\text { Procesos sur-sur horizontales. }\end{array}$ \\
\hline $\begin{array}{l}\text { Orientación con teorías de las } \\
\text { relaciones internacionales }\end{array}$ & Realismo Clásico. & $\begin{array}{l}\text { Neorrealismo, tratando de dar } \\
\text { cuenta de la interdependencia } \\
\text { compleja. }\end{array}$ \\
\hline Actores & $\begin{array}{l}\text { Participación exclusiva de los } \\
\text { Estados Nacionales soberanos. }\end{array}$ & $\begin{array}{l}\text { Los Estados Nacionales continúan } \\
\text { detentando alto protagonismo pero } \\
\text { se incorporan diversos actores no } \\
\text { estatales del sistema internacional. }\end{array}$ \\
\hline Integración económica & $\begin{array}{l}\text { Se caracterizó por un proceso } \\
\text { de integración económica hacia } \\
\text { adentro y proteccionista. }\end{array}$ & $\begin{array}{l}\text { Abierta y compatible con } \\
\text { una economía mundial } \\
\text { interdependiente. Basada en } \\
\text { acuerdos comerciales (cerca de } \\
140 \text { acuerdos entre } 1990 \text { y } 2005 \text { en } \\
\text { américa latina) (Sanahuja, 2009). }\end{array}$ \\
\hline Objetivos & $\begin{array}{l}\text { Objetivos específicos: seguridad o de } \\
\text { tipo económico. }\end{array}$ & $\begin{array}{l}\text { Multidimensional acorde a la nueva } \\
\text { agenda internacional y retos de un } \\
\text { entorno globalizado. }\end{array}$ \\
\hline
\end{tabular}

Fuente: construcción propia con base en (De Lombaerde, Kochi, \& Briceño, pág. 5)

Por lo tanto, como se muestra en el cuadro anterior, se puede señalar que el nuevo regionalismo adquiere un carácter político, sin abandonar, claro está, la orientación económica del regionalismo tradicional. Igualmente, es preciso destacar que éste se asume como una estrategia de política exterior y de integración para promover el desarrollo y mejorar la inserción internacional (Sanahuja, 2009).

Por otra parte, se marca como una diferencia sustancial el hecho de que los esquemas regionales de integración que se inscriben en el nuevo regionalismo, dejan de estar estrictamente orientados a hacer más eficientes los intercambios económicos, ya que el nivel de los compromisos asumidos por los gobiernos mediante este tipo de arreglos novedosos propicia la cooperación en áreas políticas o sociales (Gambrill \& Ruiz Nápoles, 2006, pág. 24) para atender problemáticas comunes. Sumado a lo anterior, se identifica también que el nuevo regionalismo propende por una inserción a las cadenas de valor global, principalmente por medio de atracción de inversiones, para de esta manera favorecer una mayor competitividad de la industria a nivel global, dejando a un lado la búsqueda exclusiva del aumento de la ganancia 
derivada de un mayor flujo comercial en el seno del bloque (Diaz, 2010).

En este orden de ideas, Daniela Perrotta señala que de acuerdo con el Banco Interamericano de Desarrollo (BID) el nuevo regionalismo posee como objetivos los siguientes: fortalecimiento de las reformas económicas estructurales, transformación económica gracias a nuevas oportunidades de exportación y diversificación de mercados, atracción de inversiones extranjeras, promoción de la democracia, desarme y aumento del poder negociador en foros mundiales y, finalmente, cooperación regional funcional (2013).

No obstante, en lo últimos años, en la región latinoamericana se han creado esquemas integracionistas que tienen características particulares que no encajan en las anteriormente descritas, tal es el caso de iniciativas como Unasur y el ALBA principalmente. A estas iniciativas José Antonio Sanahuja las denomina como integración post liberal; en tanto son aquellas surgidas principalmente desde mediados de la década del 2000, bajo un contexto regional muy diferente al vivido en la década de los 90. Un contexto caracterizado a grandes rasgos por el distanciamiento de los Estados Unidos de la región a causa de su concentración geopolítica en los conflictos de oriente medio, también con la llegada al poder en varios de los países latinoamericanos de gobiernos denominados de izquierda y por otra parte, en estrecha relación al aspecto anterior, el surgimiento de liderazgos regionales como Venezuela y principalmente Brasil, quienes incorporan al interior de sus respectivas políticas exteriores, la promoción y creación de estos tipos de integración regional. Sanahuja las describe con las siguientes características:
- La primacía de la agenda política y una menor atención a la agenda económica y comercial, lo que no es ajeno a la llegada al poder de distintos gobiernos de izquierda, al tono marcadamente nacionalista de estos gobiernos y a los intentos de ejercer un mayor liderazgo en la región por parte de algunos países, en particular Venezuela y Brasil.

- El retorno de "agenda de desarrollo", en el marco de las agendas económicas del "post-consenso de Washington", con políticas que pretenden distanciarse de las estrategias del regionalismo abierto, centradas en la liberalización comercial.

- Un mayor papel de los actores estatales, frente al protagonismo de los actores privados y las fuerzas del mercado del modelo anterior.

- Un énfasis mayor en la agenda "positiva" de la integración, centrada en la creación de instituciones y políticas comunes y en una cooperación más intensa en ámbitos no comerciales, lo que, como se indicará, ha dado lugar a la ampliación de los mecanismos de cooperación sur-sur, o la aparición de una agenda renovada de paz y seguridad.

- Mayor preocupación por las dimensiones sociales y las asimetrías en cuanto a niveles de desarrollo, así como la vinculación entre la integración regional y la reducción de la pobreza, en un contexto político en el que la justicia social ha adquirido mayor relevancia pero en la agenda política de la región.

- Mayor preocupación por los "cuellos de botella" y las carencias de la infraestructura regional, con el objeto de mejorar la articulación de 
los mercados regionales y, al tiempo, facilitar el acceso a mercados externos.

- Más énfasis en la seguridad energética y la búsqueda de complementariedades en este campo.

- La búsqueda de fórmulas para promover una participación y la legitimación social de los procesos de integración. (Sanahuja, 2009)

Casi en paralelo al surgimiento de estas iniciativas denominadas integracionistas posliberales, después de un periodo de estancamiento, surgen en la región iniciativas con fuertes características del nuevo regionalismo y del regionalismo abierto, es precisamente la Alianza del Pacífico, la cual, se autodenomina como un esquema de integración profunda.

La integración profunda, por su parte, describe el aumento de la interdependencia regida por las fuerzas de la internacionalización y la consecuente reducción de la brecha entre políticas nacionales. Sus derivaciones prácticas consisten en una lista de requisitos que las políticas regionales deben satisfacer para calificar como elementos de profundización de la integración: menores diferencias en estándar y producción regionales; la existencia de mecanismos gubernamentales estables; la eliminación de las barreras arancelarias y para arancelarias de los mercados importantes, y la supresión de trabas al comercio de servicios. (De la Reza, 2003)

La integración profunda se presenta de cara a lo que Richard Baldwin denomina comercio del siglo XXI, con una marcada diferencia del aquel surgido en el siglo pasado. El comercio del siglo XX y del siglo XXI son dos conceptos diferentes. Para ponerlo en términos simples, el principal - y casi único propósito- del comercio del siglo XX era producir bienes en un país para después venderlos en otro. En este sentido, el comercio internacional se reducía a bienes que cruzaban las fronteras de los países. En el comercio del siglo XXI, sin embargo, se trata de bienes que son producidos en varios o muchos países para después ser exportados. El viejo paradigma de la coordinación global de la posguerra basado en la integración superficial se ha quebrado, y ha surgido un nuevo paradigma que impulsa a los Estados-nación hacia lo que se ha llamado una integración profunda, que toca a las políticas y regulaciones detrás de las fronteras (Arellanes, 2004, pág. 88). Es así como el comercio del siglo XXI no sólo incluye al del siglo XX sino además flujos transfronterizos más complejos relacionados con redes de producción internacionales. Incluye también el comercio de bienes intermedios, servicios, ideas, know-how, capital y personas (Baldwin, 2014).

Por lo tanto, los esquemas integracionistas denominados como profundos, lo hacen con el fin de marcar la diferencia con respecto al comercio del siglo pasado y también, del viejo regionalismo y en cierta medida del nuevo. Son esquemas de integración, que no abandonan su clara orientación liberal y aperturista, pero nacen bajo las características del comercio actual y de los escenarios planeados por la globalización económica. La globalización liberal ha puesto a competir a los Estados, y estos emplean el mecanismo de la integración como una forma de hacer cara a este reto, el cual sobrepasa la simple reducción arancelaria, si no que incluye aspectos 
profundos. La integración profunda parte de la base lograda tanto del viejo como del nuevo regionalismo, pero supera a estos dos. Una vez reducidos los aranceles, algunos se mantienen altos por ser propios de sectores vulnerables o estratégicos, quedan cuestiones pendientes para dinamizar el comercio y lograr la inserción de las economías a las Cadenas Globales de Valor (CGV).

La firma del Protocolo Adicional del Acuerdo Marco en Cartagena en febrero de 2014, está compuesto por 19 capítulos, incluye temas de nueva generación (facilitación del comercio y cooperación aduanera, obstáculos técnicos al comercio y comercio electrónico). Según None al interior de la Alianza del Pacifico, el concepto de integración profunda cobra sentido y pertinencia por medio de los compromisos de libre movilidad de bienes y servicios (financieros, marítimos, telecomunicaciones y comercio electrónico), en las acciones a favor de la libre circulación de personas en calidad de negocios, estudiantes, turistas y de capitales por medio del Mercado Integrado Latinoamericano, MILA (None, 2014).

En este sentido, se puede considerar que la integración profunda es todo un proceso de integración que pretende ir más allá que los tradicionales Tratados de Libre Comercio y de los acuerdos comerciales; es decir, busca superar la dimensión economicista, pero sin olvidarla, todo lo contario, complementarla incluyendo aspectos más amplios como la cooperación internacional. Por lo tanto, no existe un modelo de integración profunda ya que estos esquemas se construyen así mismos y van tomando identidad propia.

Cuando los acuerdos sobrepasan los ámbitos comerciales e incluyen aspectos como de cooperación, éste empieza a tomar tintes de integración profunda, es decir, se asumen compromisos en diferentes materias. Los acuerdos de libre comercio no constituyen por sí mismos la creación de institucionalidad sólida y con objetivos claramente establecidos.

Recapitulando, surge la pregunta acerca de considerar la Alianza del Pacífico como promotora de una nueva forma de integración regional. La caracterización anterior, permite responder a esta pregunta teniendo en cuenta que la Alianza del Pacífico se presenta como una nueva etapa del nuevo regionalismo abierto, lo cual se podría denominar como regionalismo abierto renovado. Conservar en esencia aspectos propios del nuevo regionalismo tales como: 1) la integración económica como columna vertebral, abierta y compatible con una economía mundial interdependiente, 2) los objetivos de carácter multidimensional acordes a la nueva agenda internacional y retos de un entorno globalizado y 3 ) los actores, aunque se debe tener en cuenta que si bien los Estados Nacionales continúan detentando alto protagonismo es preciso destacar que en el sistema internacional se incorporan diversos actores no estatales, principalmente el sector empresarial y comercial.

Si bien la Alianza del Pacífico, añade características propias como la carencia de una institucionalidad permanente y la conformación de una serie de áreas de trabajo (grupos técnicos) que van desde lo educativo hasta lo financiero ${ }^{1}$, también

\footnotetext{
Los grupos técnicos abracarían: comercio e integración, compras públicas, servicio de capitales, propiedad intelectual, movimiento de personas de negocios y facilitación para el transito migratorio, estrategia comunicacional, cooperación, Pymes, comité de expertos que analiza las propuestas del Consejo Empresarial de la Alianza del Pacífico (CEAP), transparencia fiscal internacional, turismo, asuntos institucionales y mejora regulatoria.
} 
Figura 1. Cronología de iniciativas integracionistas: 1991-2012

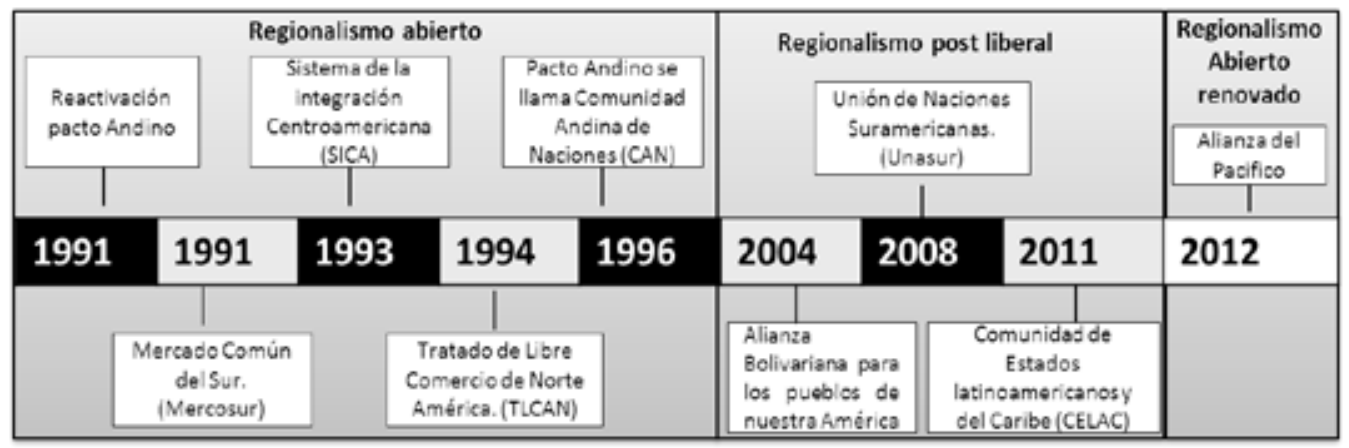

Fuente: construcción propia

vale la pena señalar que a la Alianza no es posible ubicarla en las diferentes etapas de integración clásicas (zona de libre comercio, unión aduanera, mercado común, unión económica e integración total). La A. P., es construida al margen de seguir a modo de recetario estas etapas, ya que sin duda alguna la idea es consolidar una zona de libre comercio partiendo de la armonización de los tratados de libre comercio bilaterales entre sus miembros, cosa que se logra con el protocolo adicional al acuerdo marco, de ninguna manera puede ni pretende constituirse en unión aduanera aunque tiene rasgos, no suficientes de mercado común y libre movilidad de capitales. No obstante, incluye aspectos propios como los abordados en los grupos técnicos especializados.

En la anterior figura, se propone una cronología que permite visualizar los más relevantes esquemas de integración, tanto por época de surgimiento como por sus caracteristicas. Cada uno de ellos se inscriben en cada una de las categorías de regionalismo: abierto, posliberal o abierto renovado. Claramente, la Alianza del Pacífico se incluye en la última etapa.

\section{Características Comerciales de la Alianza del Pacífico}

Tres aspectos principales económicocomerciales, comparten los países fundadores de la Alianza del Pacífico: poseer políticas comerciales aperturistas, reconocer la importancia estratégica de penetrar los mercados del Asia-Pacífico y poseer en los últimos años indicadores macroeconómicos estables así como ritmos de crecimiento por encima del promedio mundial y regional. De acuerdo con el BBVA, la Alianza en su conjunto crecerá un promedio de 4,5\% en los próximos diez años.

En este apartado se pretende realizar un análisis exploratorio basado en indicadores de comercio, haciendo énfasis en el reconocimiento de la situación colombiana en relación con el contexto de la Alianza.

Por ahora, con contadas excepciones, atrás han quedado los periodos en donde América Latina se caracterizaba a nivel internacional por altas inflaciones, tasas de crecimiento bajas, altas tasas de interés y de endeudamiento que no hacían más que rezagar cada vez más a la región en el contexto mundial. Son variados los motivos del buen 
comportamiento económico de la región, en este sentido la Comisión económica para América Latina (CEPAL), destaca:

Mejores condiciones financieras han permitido a los países mejorar el perfil de la deuda, tanto en términos de plazos como de tasas de interés, e incrementar al mismo tiempo la proporción de la deuda denominada en moneda nacional [...] la conjunción de menores tasas de interés internacionales, mejores términos de intercambio y más remesas se han traducido en una tasa de crecimiento del ingreso nacional a precios constantes superior a la del PIB [...] el coeficiente de formación bruta de capital/PIB registra actualmente el nivel más elevado de los últimos 25 años (2008).

El buen comportamiento económico también se ha expresado en un mayor dinamismo del comercio exterior, de acuerdo con la CEPAL, entre 2001 y 2013, las exportaciones de América Latina y el Caribe crecieron, en promedio, $10,1 \%$ anual, es decir, el crecimiento de las exportaciones podría doblar o triplicar el crecimiento de toda la economía (Galindo, 2014). Incluso las exportaciones de los países de la A.P. han crecido de forma sostenida en los últimos años, por encima del promedio Latinoamericano.

La gráfica 1, permite apreciar que las exportaciones de América Latina en conjunto han crecido gradualmente en los últimos años. En el año 2010 registraron un total de USD 857.863 millones y en el año 2013 fueron de USD 1.321.616 miles de millones, registrando un crecimiento de $54 \%$ y un promedio anual de $13 \%$. No obstante, sobresale que los cuatro miembros de la Alianza del Pacifico, años tras año, aportan más de la mitad de las exportaciones totales de la región latinoamericana; un 52\% para el año 2013.

Gráfica 1. Exportaciones Alianza del Pacífico vs. resto de América Latina² (2010-2013) en Millones de USD (FOB)

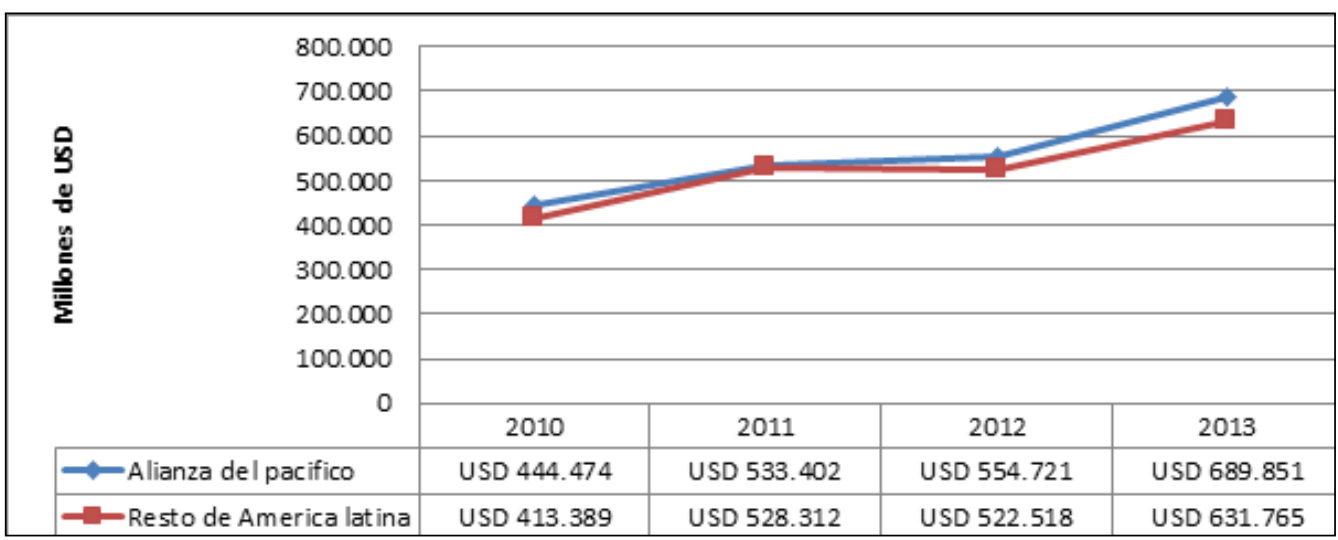

Fuente: construcción propia con datos de la (CEPAL, 2014)

2 Los países de América Latina que se tuvieron en cuenta para hacer esta sumatoria son: Argentina, Bolivia, Brasil, Costa Rica, Cuba, Ecuador, El Salvador, Guatemala, Honduras, México, Nicaragua, Panamá, Paraguay, República Dominicana, Uruguay, Venezuela. 
Lo anterior en términos poblacionales se traduce en que aproximadamente a 217 millones de habitantes corresponde el $52 \%$ del comercio de la región, versus un $48 \%$ correspondiente a 375 millones de habitantes, lo cual evidencia un claro adelanto de la Alianza en términos de exportaciones alrededor del mundo frente al resto de América Latina.

Tal diferencia en términos de exportaciones no es casual, ya que la inserción de México, Colombia, Perú y Chile a la economía mundial ha estado signada por una aceptación de la liberalización económica a partir de la eliminación de trabas o restricciones a la circulación de capitales y servicios. Como lo señala Actis "la apertura hacia la globalización económica es sin lugar a dudas un rasgo compartido" (Actis, 2014, pág. 75), previo a la creación de la Alianza, que incluso motivó a estos cuatro países para su conformación. Es decir, ha estado presente una clara orientación y apuesta hacia la internacionalización de las economias respectivas, por medio de la firma de acuerdos comerciales de diferente naturaleza, sea del orden de los Tratatados de Libre Comercio, Acuerdo de Alcance Parcial, Bloque Regional o Acuerdo Preferencial.

En este orden de ideas, la gráfica 2 permite evidenciar el buen comportamiento que han mantenido las exportaciones en la totalidad de los miembros de la A.P. durante el periodo analizado (2006-2013). En términos de crecimiento porcentual destacan los casos de Perú y Colombia. En el primero de estos, las exportaciones crecieron cerca del $75 \%$ en 7 años y para el caso de Colombia crecieron un $145 \%$ en el mismo lapso. Para estos dos países, buena parte de su crecimiento en exportaciones, sin desconocer los esfuerzos políticos aperturistas y de búsqueda de nuevas mercados, se debió en buena medida al buen ciclo en los precios de las materias primas y para el caso de Perú y Chile, Colombia y México en menor medida, la creciente demanda de estos productos por parte de China.

Gráfica 2. Exportaciones totales de países de la Alianza (2006-2013)

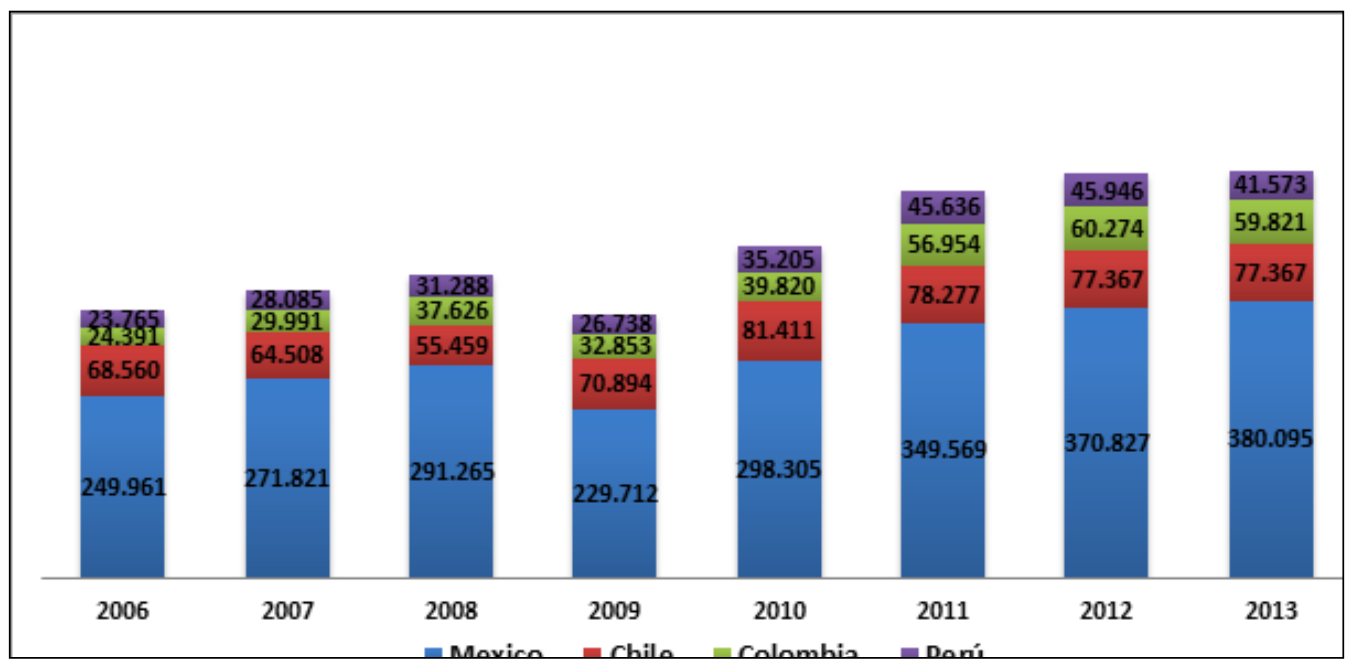

Fuente: construcción propia con base en datos de Agencias Oficiales 
China importa fundamentalmente materias primas de América Latina, y a diferencia de Europa y Estados Unidos que importan una base más diversificada de materias primas, el grueso de las importaciones Chinas son metales y minerales [...] su participación en la demanda global de hierro, cobre, aluminio y carbón se estima cerca al $60 \%$, $40 \%, 42 \%$ y $45 \%$, respectivamente (CEPAL, 2014).

Sobresale el año 2009 el cual se caracterizó por una importante caída del comercio mundial como consecuencia de la crisis financiera internacional. Esto no fue ajeno a los cuatro miembros de la Alianza, sin embargo, en el año 2010 lograron retomar los ritmos previos a la crisis. En conjunto, las exportaciones de la A.P. presentaron un crecimiento del $53 \%$ o un $7 \%$ en promedio anual.

Tabla 1. Composición de las exportaciones de la Alianza del Pacífico en (\%)

\begin{tabular}{|c|c|c|}
\hline País & $\begin{array}{c}\text { No } \\
\text { Mineras }\end{array}$ & $\begin{array}{c}\text { Mineras y } \\
\text { petróleo }\end{array}$ \\
\hline Chile & 41,5 & 58,5 \\
\hline Perú & 35 & 65 \\
\hline Colombia & 46 & 54 \\
\hline México & 85,8 & 14 \\
\hline
\end{tabular}

Fuente: construcción propia con datos de agencias oficiales
Al observar la composición de las exportaciones de los países miembros de la A.P, se evidencia la alta participación del sector minero; incluyendo el sector petróleo, en tres de los cuatro países. Tanto en Chile, Colombia, Perú este tipo de exportaciones sobrepasa el $50 \%$, registrando un $65 \%$ en el país Inca. Otra es la realidad de México, en donde, si bien es importante el sector minero-petrolero, su participación en el año 2013 fue cercano al $15 \%$ en la composición de las exportaciones del país azteca, mientras que el sector manufacturero fue de un $85 \%$ (Instituto Nacional de Estadística y Geografía, 2014).

Ahora bien, analizar el comportamiento del comercio exterior de un Estado bajo la luz exclusivamente de los exportaciones es parcializado, por lo tanto el gráfico 3 muestra la relación existente entre exportaciones e importaciones en relación con el PIB, en el periodo de tiempo (2010-2013) para cada uno de los países de la A.P. Es evidente el rezago de Colombia en esta relación, en donde tanto las exportaciones como las importaciones se encuentran muy por debajo del promedio tanto de la alianza como de la región. Se torna más preocupante la situación de Colombia cuando se comprueba que el $75 \%$ de las exportaciones de este país para el 2013 correspondieron sólo a dos tipos de productos: mineros y café, lo que se traduce en una alta dependencia exportadora de pocos productos y también una marginal participación industrial y de bienes con valor agregado. 
Gráfica 3. Relación entre exportaciones e importaciones de bienes y servicios como porcentaje de PIB (2010-2013)

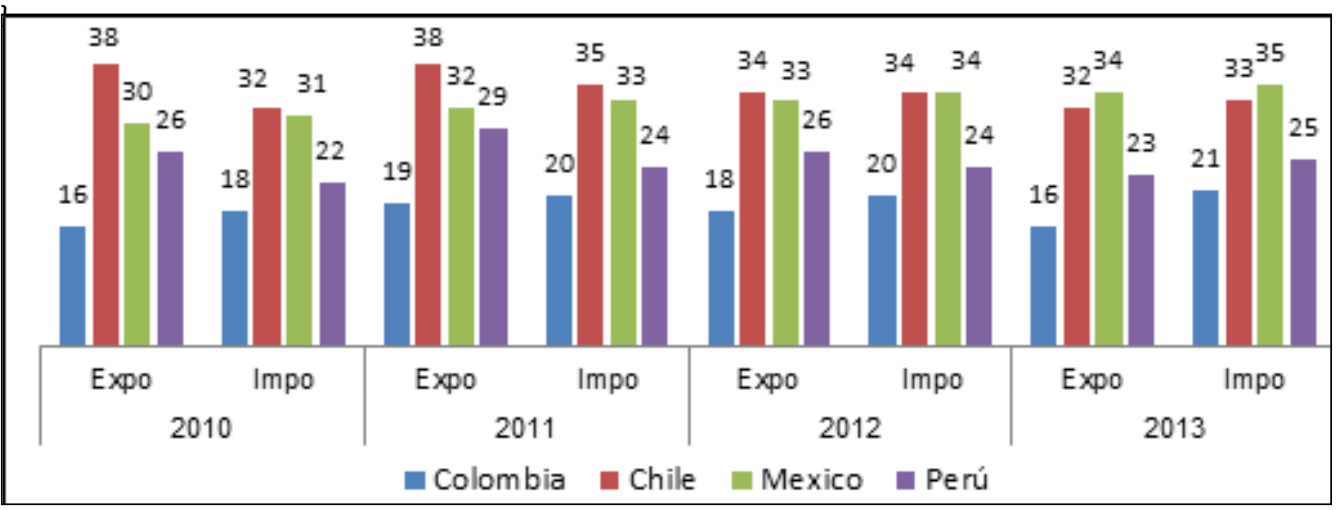

Fuente: construcción propia con base a datos del Banco Mundial

Si bien, la participación de Colombia en el comercio internacional ha mejorado en los últimos años, aún es notorio un rezago importante cuando miramos el contexto de la Alianza del Pacífico y de otras importantes economías de la región. La relación entre el valor total de la balanza comercial de cada país con respecto a su población, permite determinar el grado de apertura en comercio exterior del país en específico y comparándolo con otros. Lo arrojado por la gráfica 4 es la reafirmación de lo que se evidencia en la gráfica 3; lo cerrada que aún permanece la economía colombiana en términos de comercio exterior ubicándose en el último lugar entre los países de la Alianza del Pacífico.

3 Las exportaciones de bienes y servicios representan el valor de todos los bienes y demás servicios de mercado prestados al resto del mundo. Incluyen el valor de las mercaderías, fletes, seguros, transporte, viajes, regalías, tarifas de licencia y otros servicios tales como los relativos a las comunicaciones, la construcción, los servicios financieros, los informativos, los empresariales, los personales y los del Gobierno. Excluyen la remuneración de los empleados y los ingresos por inversiones (anteriormente denominados servicios de los factores), como también los pagos de transferencias. Las importaciones de bienes y servicios representan el valor de todos los bienes y otros servicios de mercado recibidos del resto del mundo. Incluyen el valor de las mercaderías, fletes, seguros, transporte, viajes, regalías, tarifas de licencia y otros servicios tales como los relativos a las comunicaciones, la construcción, los servicios financieros, los informativos, los empresariales, los personales y los del Gobierno. Excluyen la remuneración de los empleados y los ingresos por inversiones (anteriormente denominados servicios de los factores), como también los pagos de transferencias (Banco Mundial, 2014). 
Gráfico 4. Exportaciones per-cápita de cada país de la Alianza del Pacífico en miles de dólares durante el año 2013

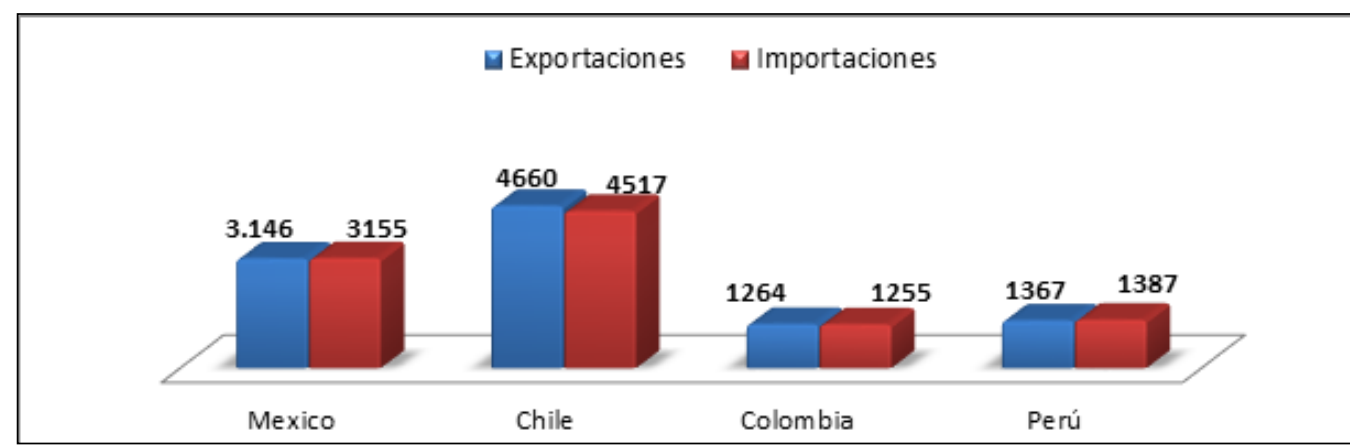

Fuente: construcción propia con base en datos de Agencias Oficiales

En promedio las exportaciones percápita de la A.P. son de USD 2609 y las importaciones USD 2579. Si bien, Colombia desde hace algunos años ha manejado una política exterior comercial orientada en la diversificación de mercados, empleando como estrategia principal la firma de acuerdos comerciales, destacándose la entrada en vigencia del Tratado de Libre Comercio con los Estados Unidos y el Acuerdo de Asociación Económica con la Unión Europea, aún no es prematuro evidenciar los impactos.

Tabla 2. Acuerdos comerciales de los miembros de la alianza del pacifico.

\begin{tabular}{|c|c|c|c|}
\hline Colombia & Chile & México & Perú \\
\hline AELC* & $\begin{array}{c}\text { Acuerdo P4 } \\
\text { (Transpacífico) }\end{array}$ & AELC & AELC \\
\hline $\mathrm{ALADI}^{* *}$ & AELC & ALADI & ALADI \\
\hline Canadá & ALADI & Chile & CAN \\
\hline Chile & Australia & Colombia & Canadá \\
\hline México & Canadá & Costa Rica & Chile \\
\hline Triángulo del Norte & China & Israel & China \\
\hline Estados Unidos & Colombia & Japón & Estados Unidos \\
\hline SGPC ${ }^{* * *}$ & Costa Rica & El Salvador & Japón \\
\hline Unión Europea & El Salvador & Guatemala & México \\
\hline
\end{tabular}

* Asociación Europea de Libre Comercio.

** Asociación Latinoamericana de Integración.

*** Acuerdo que concede preferencias arancelarias y no arancelarias entre los países en desarrollo. 


\begin{tabular}{|c|c|c|c|}
\hline Colombia & Chile & México & Perú \\
\hline Perú & Estados Unidos & Honduras & Panamá \\
\hline CAN & Guatemala & Nicaragua & Corea del Sur \\
\hline Mercosur $^{* * * *}$ & Honduras & Perú & Singapur \\
\hline Caricom $^{* * * *}$ & India & SGPC & SGPC \\
\hline & Japón & TLCAN & Unión Europea \\
\hline & Malasia & Unión Europea & Mercosur \\
\hline & México & Mercosur & \\
\hline & Panamá & & \\
\hline & Perú & & \\
\hline & Corea del Sur & & \\
\hline & SGPC & & \\
\hline & Turquía & & \\
\hline & Unión Europea & & \\
\hline & Mercosur & & \\
\hline & Hong Kong & & \\
\hline & & & \\
\hline & & & \\
\hline
\end{tabular}

Fuente: construcción propia con base en datos de Agencias Oficiales

**** Mercado Común del Sur. ***** Comunidad del Caribe.

La cantidad de acuerdos comerciales firmados por un país da cuenta de la disposición y del interés de dicho país hacia el comercio internacional. Teniendo esto en cuenta, la tabla 2 confirma lo reflejado en las gráficas 3 y 4 en el sentido de que Colombia es el país de la Alianza del Pacífico que menos acuerdos comerciales tiene con otros países, la mayoría de ellos firmados hace menos de quince años. No se puede olvidar que durante gran parte del siglo XX Colombia ignoró la escena internacional y dio la espalda a un mundo que se conectaba con mayor facilidad gracias a los avances tecnológicos y a las políticas económicas liberales implementadas por los países desarrollados. Este tipo de actitudes hicieron que Colombia fuera conocida como el Tíbet de Suramérica, en razón de su aislamiento y desconexión del mundo exterior.

Chile, por el contrario, es el país, de los cuatro países miembros de la Alianza del Pacífico, que más acuerdos comerciales tiene firmados con otros países. Chile tiene acuerdos firmados con países con un importante poder adquisitivo como Australia, Canadá y Corea del Sur. Sin dejar de lado a China que en los últimos años ha tenido un crecimiento económico importante así como India que es un mercado atractivo.

Si bien, Colombia, al igual que buena parte de la región latinoamericana, durante décadas optó por un modelo de sustitución de importaciones de corte cepalino, no obstante bajo el marco del consenso 
de Washington y las estrategias neoliberales, el país inicia un proceso de apertura desde inicios de la década de los noventa, la Ley marco de comercio exterior, Ley 07 de 1991, fue la encargada de materializar esta apertura, al permitir una reducción unilateral de aranceles como también la eliminación de barreras no arancelarias a las importaciones y exportaciones. Proceso que se vio seriamente interrumpido y afectado, entre otros factores por la baja competitividad del país en un entorno internacional y por la agudización y desbordamiento del conflicto armado de la mano del flagelo del narcotráfico.
La década del 2000, se inicia con un nuevo intento de internacionalizar la economía, esta vez, no por eliminación unilateral, sino principalmente por medio de los Tratados de Libre Comercio, como uno de los pilares para lograr el desarrollo económico del país. Por lo tanto, en comparación con países como México y Chile, cuya estrategia de inserción al comercio mundial basados en acuerdos comerciales, Colombia, inicia este proceso un poco tarde, lo cual explica por una parte el número de acuerdos como también el valor tanto de exportaciones como de importaciones.

Figura 2. Acuerdos Comerciales de la Alianza del Pacífico

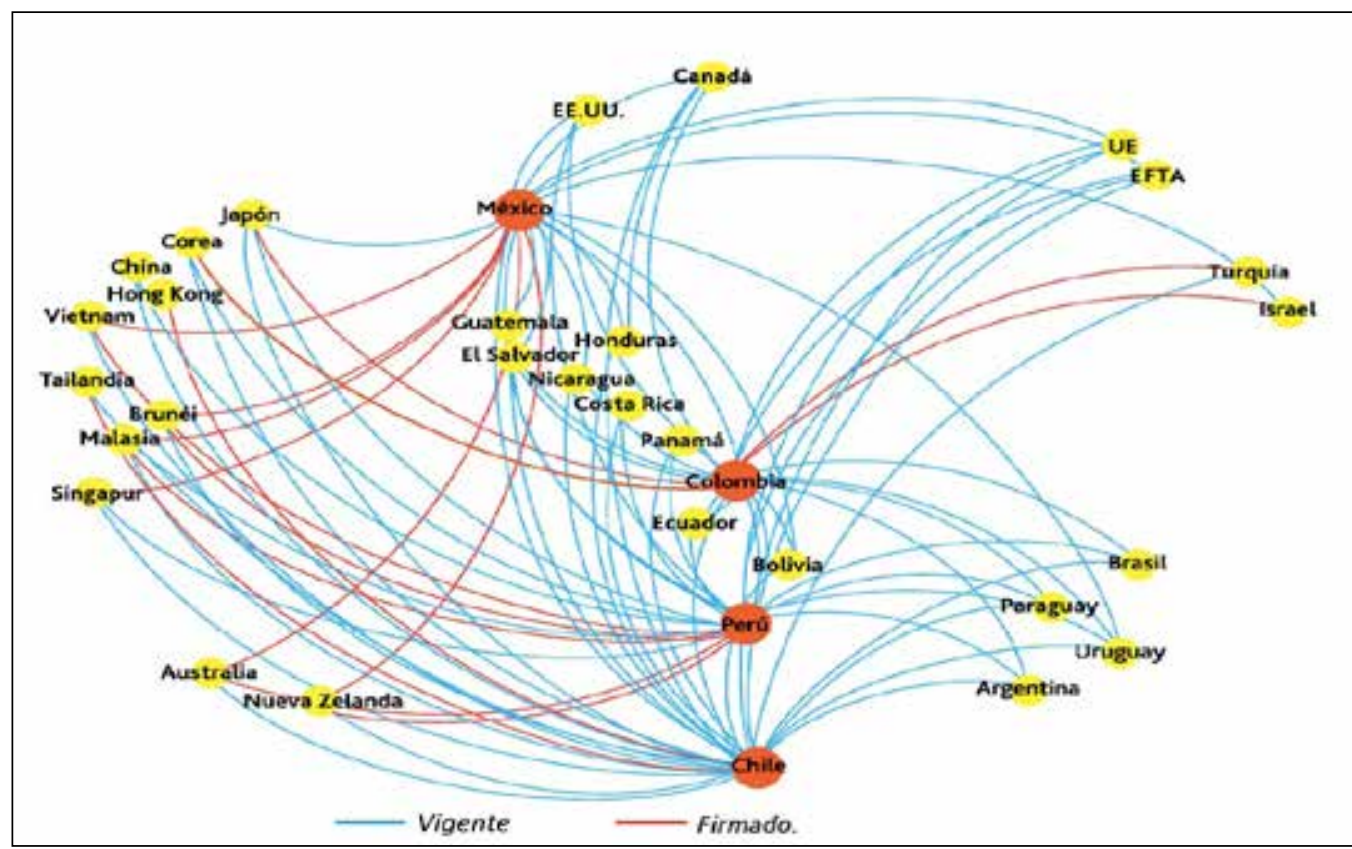

Fuente: (World Economic Forum, 2014) 
Finalmente, a modo de complemento de la tabla 2, la figura anterior, a modo gráfico detalla la compleja red de acuerdos comerciales que han logrado establecer los diferentes miembros de la A.P. en las últimas décadas. Acuerdos con países con diferentes niveles de ingresos y estructuras económicas.

\section{Colombia de cara a la Alianza del Pacíico. Retos y desafíos}

Se podría señalar que Colombia ha obtenido beneficios relativos pero oportunidades potenciales, interacción comercial con los países de la A.P., tanto antes como después de la creación de este esquema integracionista.
En este orden de ideas, la tabla 3, muestra la evolución en el presente siglo que han tenido los 10 principales socios comerciales de las exportaciones colombianas. En primer lugar, se evidencia, entre el año 2000 hasta el 2014 una disminución de la participación porcentual de estos socios. Para el año 2000 84,3\% de las exportaciones colombianas se dirigían a 10 destinos, durante el 2014 fue cerca de un 79\%, lo que muestra una pequeño aumento en la diversificación de socios comerciales generada especialmente por la entrada en vigencia de los diferentes TLC. Por otra parte, en particular con los miembros de la alianza, se observa una participación un poco marginal en las exportaciones colombianas con variaciones menores en los 15 años de observación.

Tabla 3. Participación porcentual de principales destinos de exportaciones colombianas (2000-2014)

\begin{tabular}{|c|c|c|c|c|c|c|}
\hline País & $\mathbf{2 0 0 0}$ & $\mathbf{2 0 0 5}$ & País & $\mathbf{2 0 1 1}$ & País & $\mathbf{2 0 1 4}$ \\
\hline Estados Unidos & 49,6 & 40 & Estados Unidos & 37,6 & Estados Unidos & $\mathbf{2 5 , 7}$ \\
Unión Europea & 13,8 & 13,3 & Unión Europea & 15,5 & Unión Europea & 17,2 \\
Venezuela & 9,9 & 9,9 & Chile & 3,92 & China & 10,5 \\
Ecuador & 3,5 & 6,3 & China & 3,54 & Panamá & 6,6 \\
Perú & 2,8 & 3,4 & Panamá & 3,49 & India & 5 \\
México & 1,8 & 2,9 & Ecuador & 3,4 & Venezuela & 3,6 \\
Japón & 1,8 & 1,6 & Venezuela & 3,1 & Ecuador & 3,4 \\
R. Dominicana & 0,8 & 2,8 & Aruba & 3,0 & Brasil & 3,0 \\
Suiza & 0,1 & 0,7 & Perú & 2,5 & Perú & 2,2 \\
China & 0,2 & 1,1 & Brasil & 2,4 & Chile & 1,8 \\
Total & $84,3 \%$ & $82 \%$ & & $78,45 \%$ & & $79 \%$ \\
\hline
\end{tabular}

Fuente: construcción propia con datos (Ministerio de Comercio, Industria y Turismo, 2015) 
Incluso la participación de los tres restantes miembros de la alianza ha disminuido en la presente década, a modo de ejemplo; teniendo presente que las exportaciones a México representaron un 1,2\%, para dicho año los tres países representaron un $7,8 \%$ de la exportaciones colombianas y para el año 2014, cuando hacia México se exportó un 1,7\% el total para los miembros de la alianza fue $5,7 \%$, recordando que antes de la creación de la A.P. existían diferentes mecanismos y acuerdos comerciales de Colombia con estos países. resaltar, que más del $40 \%$ de las importaciones colombianas de cada año de referencia se concentró en los dos primeros socios importadores.

Según lo anterior, se puede constatar cómo en los años 2000 y 2005 , el 49,88\% y el $42,53 \%$, respectivamente, el origen de las importaciones colombianas fueron los Estados Unidos y los países de la Unión Europea. En los años 2011 y 2014 el $40,04 \%$ y el $46,78 \%$, respectivamente, provinieron de Estados Unidos y de China, que relegó a la Unión

Tabla. Participación porcentual de principales orígenes de

importaciones colombianas (2000-2014)

\begin{tabular}{|c|c|c|c|c|c|c|c|}
\hline País & $\mathbf{2 0 0 0}$ & País & $\mathbf{2 0 0 5}$ & País & $\mathbf{2 0 1 1}$ & País & $\mathbf{2 0 1 4}^{*}$ \\
\hline Estados Unidos & 33,14 & Estados Unidos & 28,61 & Estados Unidos & 25,17 & Estados Unidos & 28,69 \\
\hline Unión Europea & 16,74 & Unión Europea & 13,92 & China & 14,87 & China & 18,09 \\
\hline Venezuela & 8,19 & México & 8,50 & Unión Europea & 13,12 & Unión Europea & 13,80 \\
\hline México & 4,70 & China & 7,39 & México & 11,35 & México & 8,21 \\
\hline Japón & 4,65 & Brasil & 6,43 & Brasil & 5,08 & Brasil & 3,88 \\
\hline Brasil & 4,31 & Venezuela & 5,87 & Argentina & 3,31 & Japón & 2,38 \\
\hline China & 2,90 & Japón & 3,31 & Japón & 2,64 & Corea del Sur & 2,27 \\
\hline Ecuador & 2,75 & Corea del Sur & 2,77 & Corea del Sur & 2,23 & Perú & 1,82 \\
\hline Canadá & 2,38 & Ecuador & 2,58 & Ecuador & 2,02 & Canadá & 1,80 \\
\hline Chile & 2,14 & Argentina & 1,81 & Perú & 1,92 & Argentina & 1,58 \\
\hline Total & $81,9 \%$ & & $81,2 \%$ & & $81,7 \%$ & & $82,5 \%$ \\
\hline
\end{tabular}

Fuente: construcción propia con datos (Ministerio de Comercio, Industria y Turismo, 2015)

* El año 2014 toma los meses de enero a noviembre.

En este orden de ideas, la Tabla 4 muestra la evolución de la participación porcentual de los diez primeros socios de las importaciones colombianas, tomando como referencia los años 2000, 2005, 2011 y 2014. En primer lugar, se puede apreciar que en cada uno de los cuatro años, más del $81 \%$ de las importaciones colombianas estuvieron concentradas en diez países. Estos diez países no siempre fueron los mismos y su participación porcentual no se distribuyó de manera simétrica. Cabe
Europea como segundo socio importador de Colombia. Vale la pena mencionar que en los cuatro ańos de referencia, Estados Unidos fue el principal origen de las importaciones colombianas, así como también el único país que ha conservado su lugar a lo largo de los años, aunque su porcentaje de participación en las importaciones 
colombianas haya decrecido de un $33,14 \%$ en el año 2000 al 28,69\% en el año 2014.

Si se analiza la participación porcentual de los miembros de la Alianza del Pacífico, se evidencia que en el año 2011, en conjunto, representaron el $14,91 \%$ del total de las importaciones colombianas, mientras que en el año 2014, representaron el $11,48 \%$; siendo México el socio importador más relevante dentro de los países de la A. P., con participaciones del $11,35 \%$ (2005) y del 8,21\% (2014), mientras que Perú y Chile sólo tuvieron participaciones residuales en dichos años.

Por lo tanto, es preciso recordar que para Colombia la Alianza del Pacífico representa un mercado de cerca de 160 millones de habitantes con una capacidad adquisitiva media-alta y en crecimiento, pero continúa siendo un mercado poco explotado por parte de Colombia, presentando oportunidades potenciales. No obstante, el aprovechamiento de este mercado radica también, en el reto que tiene Colombia de diversificar su oferta exportadora; si se remite nuevamente a la tabla 1, la cual muestra la composición de las exportaciones de los países miembros de la A. P., se puede explicar un poco el hecho de los pocos cambios evidenciados en la balanza comercial entre Colombia con Perú y Chile, en el sentido de la alta dependencia exportadora de estos tres países frente a los recursos mineros, energéticos, lo cual convierte a estos países andinos, en economías más cercanas a la competición que a la complementariedad.

Paralelamente, queda evidenciado que las exportaciones en relación con las importaciones, siguen siendo el talón de Aquiles para el país, en tanto pese a la decidida apertura económica a la que hemos asistido en los últimos años, a través de los tratados y acuerdos comerciales, el país sigue gozando de poca vocación exportadora y de capital empresarial con apuestas de internacionalización; en términos porcentuales, Colombia importa el doble de los países de la A. P. (11,48\% en 2014), de lo que exporta a estos destinos (5,7\% en 2014). Por lo tanto, lograr una relación comercial más equilibrado es uno de los principales retos para la economía colombiana en el marco de esta iniciativa integracionista.

Ahora bien, no se pueden desconocer los beneficios que tiene esta iniciativa de nuevo regionalismo renovado concebido desde su nacimiento como un proceso con poca burocracia institucional. Entre los beneficios que se destacan figuran: la inserción con el Asia Pacífico, la cooperación entre los países miembros, la eliminación de visas de viajero, la puesta en funcionamiento del Mercado Integrado Latinoamericano (MILA), la liberalización de bienes, servicios e inversiones, la proyección internacional, la cooperación en asuntos de aduana, entre otros.

Colombia debe aprovechar y potencializar todos estos beneficios con el fin de obtener una mejor cosecha en sus procesos de negociación e integración regional. Según Trujillo Acosta, una de las principales ventajas de la A. P. para Colombia es que "le facilita profundizar sus relaciones regionales sin generar tensiones con terceros países ni otros grupos regionales" sumado a "la oportunidad para construir un liderazgo sobre la generación de consensos" asumiendo un papel protagónico e influyente dentro de las relaciones internacionales.

De acuerdo con Arévalo Luna, se podría indicar que los desafíos y retos para el país están primordialmente, en el campo comercial, económico y de las relaciones internacionales, en este sentido: 
Colombia, deberá aunar esfuerzos y realizar políticas públicas adecuadas para superar su atraso en infraestructura, educación y sistemas de ciencia y tecnología y en lo concerniente al campo de las relaciones económicas internacionales, el país debe procurar una participación más activa dentro de los diversos organismos internacionales, aspecto que favorecerá el papel de Colombia como jugador global (2014, p. 171).

Algunos de los países del Asia Pacífico a los cuales se quiere llegar con la alianza son: Japón, India, China, Corea del Sur, Tailandia, por mencionar algunos, los cuales han realizado cambios estructurales en sus economías conforme a la dinámica de apertura económica a la que se han insertado. En consonancia con lo anterior, los países de la A. P. y de manera particular Colombia, debe consolidar una estructura productiva fuerte y diversificada de modo que le permita ser competitiva en una economía cada vez más exigente y globalizada, de no ser así, los beneficios seguirán siendo modestos.

Colombia encontró en el regionalismo renovado de la alianza, la ventana de oportunidad que le permite y permitirá acortar brechas para mejorar su participación en el mercado de Asia Pacífico (Tremolada, 2014), no obstante, de no acelerar y promover cambios como los citados previamente, nuestros propios socios nos seguirán desplazando.

Finalmente uno de los importantes retos que tiene Colombia, en sentido general de cara a la internacionalización de la economía es mejorar su desempeño logístico. Resulta contradictorio que la política de desarrollo de Colombia basada en la internacionalización de la economía y de la inserción a las Cadenas Globales de Valores (CGV), no han sido acompañadas por mejorías significativas en los índices internacionales, especialmente en el índice de desempeño logístico que año a año desarrolla el Banco Mundial. Luego de alcanzar el puesto 64 entre 155 países en el Logistics Performance Index de 2012 (LPI), Colombia cayó al puesto 97 entre 160 países en el 2014. Las posiciones ocupadas por Colombia en cada emisión del LPI han sido: puesto 82 en 2007, puesto 72 en 2010, puesto 64 en 2012 y puesto 97 en 2014. Luego de un notorio avance de 2007 a 2012, el país, en solo dos años, pierde prácticamente todo lo que había avanzado. Desde el punto de vista del indicador integral de desempeńo, que es el que sirve para posicionar cada nación, los valores han sido de 2,50, 2,77, 2,87 y 2,64 para 2007, 2010, 2012 y 2014 respectivamente. A manera de referencia, el máximo puntaje en el LPI de 2014 lo obtuvo Alemania con 4,12, de 5 puntos posibles. Chile, que encabeza el grupo de países latinoamericanos, obtuvo un puntaje de 3,26.

\section{Conclusiones}

Las exportaciones de América Latina en conjunto han crecido gradualmente en los últimos años, sin embargo, es de resaltar que los cuatro miembros de la Alianza del Pacífico, aportan más de la mitad de las exportaciones totales de la región latinoamericana. Esto se debe principalmente a la inserción de los cuatro países miembros en la economía mundial a través de la eliminación de las restricciones a la cirulación de personas, bienes, servicios y capitales.

Cabe resaltar el buen comportamiento que las exportaciones de los cuatro países miembros de la A.P. han mantenido 
desde el 2006. Es notorio el crecimiento porcentual de las exportaciones tanto de Perú como de Colombia. Sin embargo, es preocupante que la oferta exportadores de los cuatro países se reduce al sector minero y al sector petróleo. En Chile, Colombia y Perú este tipo de exportaciones supera el 50\%. En México, la participación del sector minero es cerca del $15 \%$ teniendo su sector manufactuero un peso importante con un $85 \%$ de la oferta exportadora del país Azteca.

Si bien es cierto que la participación de Colombia en el comercio internacional ha mejorado constantemente en los últimos años, su rezago con los demás países de la Alianza del Pacífico y de otras economías América Latina aún es considerable. Vimos cómo tanto las exportaciones como las importaciones se encuentran muy por debajo del promedio de la Alianza del Pacífico y de América Latina. Lo que es más preocupante aún, la mayor parte de la oferta exportadora de Colombia se reduce a dos tipos de productos: los mineros y a el café.

Finalmente, la Alianza del Pacifico, la cual se presenta como un nuevo regionalismo renovada o acorde con el contexto histórico e internacional particular de la región latinoamericana y especialmente de los países que hacen parte de esta alianza, se presenta como una importante ventana de oportunidades para sus miembros; en poco tiempo de existencia se pueden reconocer avances significativos para un esquema de integración, no obstante, para el caso colombiano, es preocupante el aislacionismo en términos de comercio internacional que aun vive el país, a pesar de los numerosos tratados de libre comercio firmados y vigentes en la actulidad. Lo cual demuestra que la estrategia de internacionalización de la economía colombiana no debe tener como fin la firma de este tipo de tratados, sino vistos como uno de las diversas herramientas para lograr este fin, herramientas como: promoción de exportaciones, ruedas de negocios, mejoría sustancial de la competitividad e infraestructura logística, por mencionar algunos.

\section{Bibliografía}

Actis, E. (2014). Brasil y sus pares sudamericanos de la "Alianza del Pacifico". Divergencias regionales en las opciones nacionales e internacionales. Relaciones Internacionales(46), 71-87.

Alianza del Pacífico. (17 de Junio de 2014). alianzapacifico. Recuperado de http:// alianzapacifico.net/mexico-recibe-decolombia-una-alianza-del-pacificofuerte/

Arellanes, P. E. (2004). Avances en la agenda Social del ALCA. Geoenseñanza, 9(1), 85-96.

Arevalo G. (2014). La Alianza Pacífico: geopolítica e integración económica. Revista Via Iuris, Número 16, enerojunio, pp. 159-172

Baldwin, R. (2014). Multilateralising 21st century Regionalism. Paris: OCDE.

Banco de la República. (10 de julio de 2011). www.banrep.gov.co. Revisado el 15 de octubre de 2011. Recuperado de: http:// www.banrep.gov.co/estad/Balanzapagos/III-Trim-2011.pdf

Banco de la República. Series estadísticas. (24 de 07 de 2010). www.banrep.gov.co. Revisado el 24 de 07 de 2010. Recuperado de: http://discoverer.banrep.gov.co/discoverer/app/ grid?event=displayData\&stateStr= eNrtU01v2zAM/TOyL0YCSTHS9eBbu50KDF3vgSzJthJ/ 
VVItO79\$1JQs2YatwLpDDwUMPoqiH/ kEErmDaSxKaC6mnTCACd0\$t2Y3J5 Rsks3dEzMsubmn\$FGOWhrZW2bV FENCtgEfpOZMDMFfBfv0\$LAOzp Wh26oDwrlrTyU6KIHfVOLH

Banco Mundial. (20 de Abril de 2014). Banco Mundial. Recuperado de: http://datos. bancomundial.org/indicador/NE.EXP. GNFS.ZS

BANCOLDEX. (23 de 06 de 2007). http://www.bancoldex.com. Revisado el 08/04/2010, Recuperado de: http://www. bancoldex.com/contenido/contenido. aspx?catID $=261 \&$ con ID $=586$

CEPAL. (20 de Diciembre de 2010). www. eclac.org. Revisado el 23 de Marzo de 2011. Recuperado de: http://www.eclac. org/cgi-bin/getProd.asp?xml=/publicaciones/xml/8/41898/P41898.xml\&xsl=/ $\mathrm{de} / \mathrm{tpl} / \mathrm{p} 9 \mathrm{f} . \mathrm{xs} l \& b a s e=/ \mathrm{tpl} /$ top-bottom.xslt CEPAL. (20 de Mayo de 2014). Comercio Internacional e Integración. Recuperado de:: http://www.cepal.org/comercio/ notas_comercio/

CEPAL. (2014). Perspectivas económicas de Amérca Latina 2014. Logística y competitividad para el desarrollo. Santiago de Chile: CEPAL.

Córdoba, J. (13 de Marzo de 2009). www. bvc.com.co. Recuperado el 24 de septiembre de 2010

De la Reza, G. (2003). El regionalismo abierto en el hemisferio occidental. Análisis Económico, 297-312.

De Lombaerde, P., Kochi, S., \& Briceño, J. (2008). Del regionalismo latinoamericano a la integración intrarregional. Madrid: siglo XXI.

Delegación de la Unión Europea en Colombia. (20 de 10 de 2013). Delegación de la Unión Europea en Colombia. Recuperado de: http://eeas.europa.eu/delegations/ colombia/eu_colombia/trade_relation/ eu_association_agreement/index_es.htm
Diario La República. (07 de 11 de 2009). La República.com. Revisado el 19 de 02 de 2010. Recuperado de: http://rse. larepublica.com.co/archivos/EMPRESAS/2009-11-07/exportaciones-en-ceroascoltex_87116.php

Diaz, M. P. (2010). Pespectivas del nuevo regionalismo latinoamericano desde el enfoque de los acuerdos "sur-norte": analisis de la experiencia mexicana. Madrid: Universidad Complutense de Madrid.

Fedesarrollo. (2011). Perspectivas Económicas 2011-2012. Conferencias ANIF (pág. 44). Medellín: ANIF.

FMI. (2011). Perspectivas Económicas Globales. Washington.

Galindo, M. (2014). América Latina: ¿Amenaza? ¿oportunidad? Madrid: Debate.

Gambrill, M., \& Ruiz Nápoles, P. (2006). Procesos de Integración en las Americas. Mexico: Cisan.

Instituto Mexicano de Contadores Públicos. (2010). El dinero de hoy: dinero de plástico. Evolución, riesgos y ventajas . México.

Instituto Nacional de Estadistica y Geografía. (15 de Junio de 2014). Inegi. Recuperado de: http://www.inegi.org.mx/ prod_serv/contenidos/espanol/bvinegi/ productos/continuas/economicas/exterior/mensual/ece/bcmm.pdf

Mincomercio. (19 de 02 de 2010). Mincomercio.gov.co. Revisado el 19 de 02 de 2010. Recuperado de http://www.mincomercio.gov.co/eContent/newsdetail. asp?id=7216\&IdCompany $=23$

Ministerio de Comercio, Industria y Turismo. (27 de Febrero de 2015). Mincomercio. Recuperado de: http://www.tlc.gov.co/ None, J. (Abril de 2014). Foreign Affairs. Recuperado de: http://revistafal.com/laalianza-del-pacifico-tres-anos-despues/ Periódico Portafolio. (18 de Febrero de 2010). Protafolio.com. Revisado el 19 de Febrero 
de 2010. Recuperado de: http://www. portafolio.com.co/opinion/editorial/ ARTICULO-WEB-NOTA_INTERIOR_PORTA-7056167.html

Perrotta, D. (2013). La integración regional como objeto de estudio. De las teorías tradicionales a los enfoques actuales. En E. LLenderrozas, Relaciones Internacionales: Teorías y Debates. Buenos Aires : Eudeba, pp. 197-252.

Sanahuja, J. A. (2009). del "regionalismo abierto al "regionalismo post-liberal", crisis y cambio en la integración regional en América Latina. En L. Martínez, P. Lázaro, \& V. Mariana, Caribe, Anuario de la integración Regional de
América Latina y el Gran Caribe (págs. 11-54). Buenos Aires: Coordinadora Regional de Investigaciones Econónomicas y Sociales.

Tremolada E. (2014) ¿La Alianza del Pacífico facilita la inserción de Colombia en la región Asia-Pacífico?. Papel Político, Julio-Diciembre, 721-752.

Trujillo Acosta, Iván Alejandro. (2014). Sobre las ventajas de la alianza del pacífico para colombia. Prolegómenos. Derechos y Valores, XVII Enero-Junio, 159-172.

World Economic Forum. (2014). Enabling Trade from valuation to action. Ginebra: World Economic Forum. 